JOURNAL DE PHYSIQUE IV

Colloque C6, supplément au Journal de Physique III, Vol. 1, décembre 1991

C6-21

\title{
ANALYSIS OF THE RECOMBINATION VELOCITY AND OF THE EBIC AND CATHODOLUMINESCENCE CONTRAST AT A DISLOCATION
}

\author{
R.J. TARENTO and Y. MARFAING* \\ Laboratoire de Physique des Matériaux, CNRS, 1 Place A. Briand, F-92195 Meudon Cedex, \\ France \\ *Laboratoire de Physique des Solides de Bellevue, CNRS, 1 Place A. Briand, F-92195 Meudon \\ Cedex, France
}

It is known that the presence of dislocations has a strong influence on the properties of semiconductor devices, by causing excess recombination and leakage currents. These effects originate in the existence of localized electron states associated with dislocation dangling bonds, so that characterization of those localized states is an important task.

In this respect the EBIC and cathodoluminescence contrast techniques deserve much attention because they yield local information on the recombination activity of defects. However a physical analysis of the processes involved is needed in order to deduce meaningful quantities.

In a first step we have analysed the recombination processes on a linear dislocation isolated in an uniformly excited semiconductor. The dislocation is treated within the Read model. The procedure includes several aspects : non-rigid displacement of the dislocation energy levels, bending of the minority-carrier quasi-Fermi level across the dislocation space charge and in the external quasineutral region. The dislocation energy states are considered to be uniformly distributed in a half-filled band (in neutral state).

The level occupation is controlled by the Hall-Shockley-Read mechanism. Under these conditions a self-consistent calculation yields the values of the barrier height and of the effective recombination velocity as a function of various parameters : linear density of localized states, recombination cross section, properties of the bulk material (doping, mobility, lifetime) and excitation level in the bulk.

In a second step, these results have been used to express the EBIC and cathodoluminescence (CL) contrasts near a dislocation perpendicular to the collecting or observation surface. Low excitation level has been assumed so that the recombination velocity has a (maximum) constant value along the dislocation line. The three dimensional continuity equation for diffusion and recombination has been solved for an electron beam set at some distance from the dislocation. Numerical applications to $\mathrm{Si}$ (EBIC) and GaAs (CL) lead to a contrast magnitude in agreement with experiments. 